\title{
Síndrome do Túnel Cárpico: Revisão da Literatura
}

\section{Carpal Tunnel Syndrome: A Literature Review}

Jaime Oliveira1*

\section{RESUMO}

A síndrome do túnel cárpico (STC) afeta 3\% da população e deve-se à compressão do nervo mediano no túnel cárpico. Este artigo pretende rever a evidência científica sobre a STC.

Realizou-se uma revisão narrativa. Identificaram-se 161 artigos na pesquisa e incluíram-se na revisão dados referentes a 26 referências bibliográficas. A STC afeta mais as mulheres. $O$ diagnóstico é clínico, sendo os sintomas clássicos dor, disestesias e/ou parestesias na região palmar, desde o polegar até à face radial do anelar. Quando positivas, as manobras provocatórias aumentam a suspeita diagnóstica. Os testes eletrodiagnósticos são importantes para confirmação diagnóstica, estratificação da gravidade e definição do tratamento. Perante doença ligeira/moderada, inicialmente recomenda-se tratamento conservador. Perante quadros moderados/graves, recomenda-se cirurgia.

A STC é incapacitante. Perante sintomas sugestivos, deve-se equacionar este diagnóstico e realizar provas provocatórias. Os testes eletrodiagnósticos são importantes para diagnóstico e definição da terapêutica.

PALAVRAS-CHAVE: Eletrodiagnóstico; Síndrome do Túnel Cárpico/diagnóstico; Síndrome do Túnel Cárpico/tratamento 


\section{ABSTRACT}

Carpal tunnel syndrome (CTS) affects 3\% of the population and is due to compression of median nerve in the carpal tunnel. This article aims to review the scientific evidence about CTS.

A narrative review was carried out. One hundred and sixty one articles were identified in the research and data referring to 26 bibliographic references were included in the review. CTS affects women more. The diagnosis is clinical. Classical symptoms are pain, dysesthesias and/or paresthesias in the palmar region, from the thumb to the radial surface of annular. When positive, provocative manoeuvres increase diagnostic suspicion. Electrodiagnostic tests are important for diagnostic confirmation, severity stratification and treatment definition. If the disease is mild/ moderate, conservative treatment is initially recommended. In moderate/severe cases, surgery is recommended.

CTS is disabling. When there are symptoms, this diagnosis must be considered and provocative tests performed. Electrodiagnostic tests are important for diagnosis and definition of therapy.

KEYWORDS: Carpal Tunnel Syndrome/diagnosis; Carpal Tunnel Syndrome/therapy; Electrodiagnosis

\section{INTRODUÇÃO}

A síndrome do túnel cárpico (STC) refere-se ao conjunto de sinais e sintomas causados pela compressão do nervo mediano ao longo do seu trajeto no túnel cárpico. ${ }^{1-4} \mathrm{Ge}-$ ralmente, os doentes apresentam dor, disestesias e/ou parestesias no território do nervo mediano, afetando a qualidade de vida. ${ }^{1,3,5}$

Esta patologia afeta aproximadamente 3\% da população geral, ${ }^{6}$ sendo avaliada frequentemente nos cuidados de saúde primários. ${ }^{3}$ A prevalência, impacto na qualidade de vida e custos que lhe estão associados (seja pelo absentismo laboral, seja pelos recursos de saúde alocados à sua abordagem) implicam que a STC seja alvo de investigação e de revisões periódicas da evidência científica disponível. ${ }^{4}$

O objetivo deste artigo foi rever a evidência atualmente disponível sobre a STC, no que diz respeito à sua anatomia, fisiopatologia, epidemiologia, fatores de risco, manifestações clínicas, abordagem diagnóstica e terapêutica.

\section{MÉTODOS}

Este artigo consiste num trabalho de revisão narrativa.

A 31 de janeiro de 2021 realizou-se uma pesquisa, nas plataformas PubMed e UpToDate, tendo como discriminador o termo MeSH "Carpal Tunnel Syndrome". Restringiu-se a pesquisa a meta-análises e revisões sistemáticas ou narrativas, publicadas entre 01/2015 e 01/2021 e escritas em inglês, português ou espanhol.

A leitura dos títulos e resumos permitiu excluir os artigos relativos a: a) estudos de âmbito laboratorial ou animal; b) descrição pormenorizada de técnicas diagnósticas ou terapêuticas; c) outras patologias distintas da STC. De seguida procedeu-se à leitura integral de artigos que não foram excluídos no passo anterior, tendo em vista a aplicação dos critérios de exclusão previamente mencionados.

Finalmente, procedeu-se à leitura de: a) oito referências bibliográficas relevantes identificadas nos artigos previamente lidos na íntegra; b) dois livros sobre patologia reumatológica no âmbito dos cuidados de saúde primários em Portugal.

\section{RESULTADOS}

A Fig. 1 esquematiza o processo de pesquisa e seleção dos artigos incluídos nesta revisão.

Identificaram-se 161 artigos na pesquisa e incluíram-se na revisão dados referentes a 26 referências bibliográficas.

De seguida, serão apresentados os dados pertinentes no que diz respeito à anatomia, fisiopatologia, epidemiologia, fatores de risco, manifestações clínicas, abordagem diagnóstica e terapêutica da STC.

\section{ANATOMIA E FISIOPATOLOGIA}

O nervo mediano forma-se a partir do plexo braquial, através de fibras provenientes das raízes nervosas de C6, C7, C8 e T1, podendo também existir contribuição da raiz de $C 5,7,8$ Este nervo atravessa o punho no túnel cárpico (espaço anatómico limitado superiormente pelo ligamento transverso do carpo, inferiormente pela fileira proximal do carpo, lateralmente pelo tubérculo do escafoide e trapézio e medialmente pelo gancho do unciforme e pisiforme), juntamente com os tendões dos músculos flexores superficial e profundo dos dedos e do músculo flexor longo do polegar. 3,4,8-10 


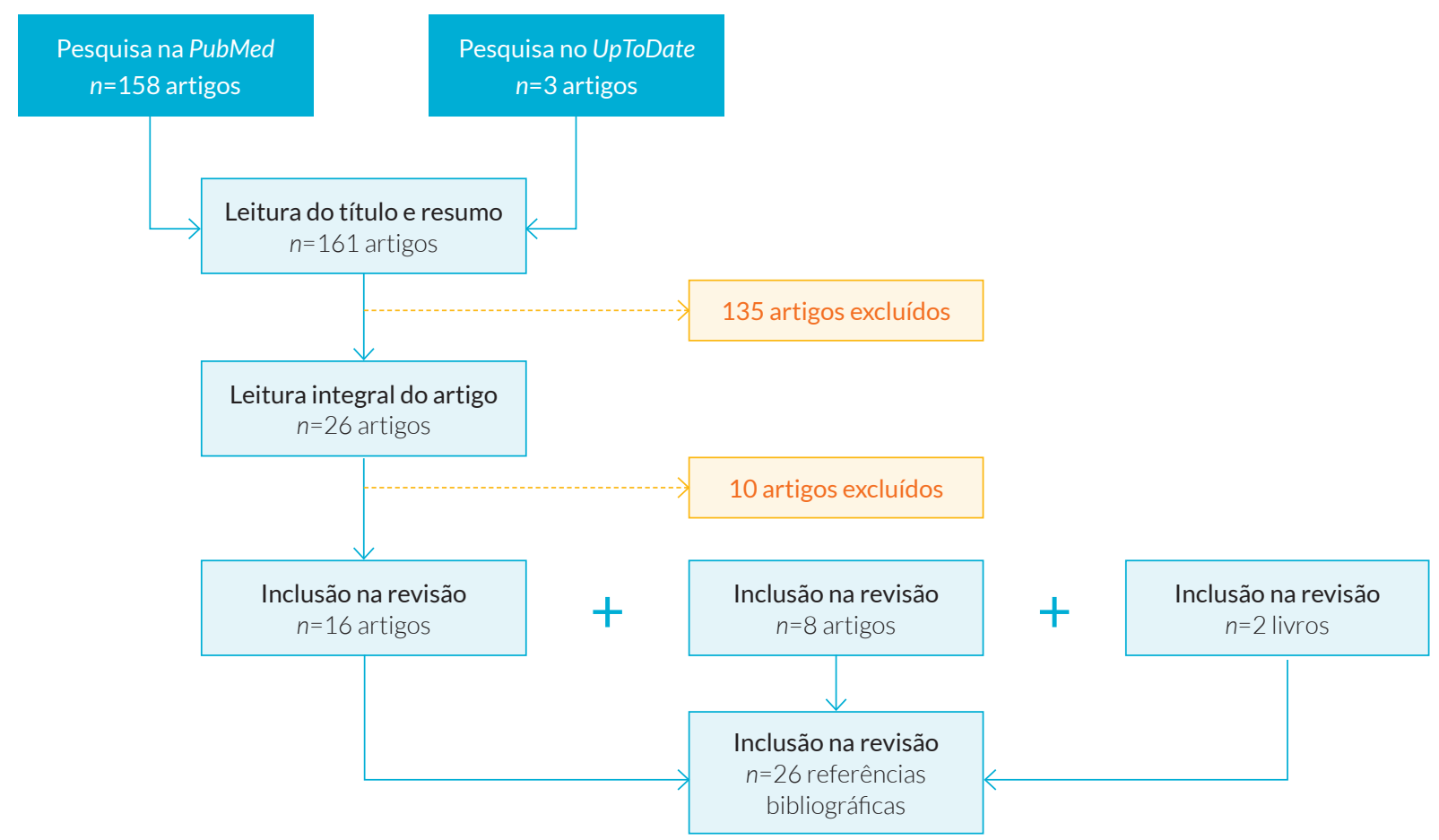

FIGURA 1. Esquematização do processo de pesquisa e seleção dos artigos incluídos nesta revisão ( $n=$ número).

Após a passagem no túnel do carpo, o nervo mediano participa na inervação motora e sensitiva da mão.7,8 Os músculos da mão que são inervados pelo nervo mediano são o abdutor curto do polegar, o flexor curto do polegar (cabeça superficial), o oponente do polegar e o primeiro e segundo lumbricais.7,8 Após a passagem pelo túnel cárpico, este nervo é responsável pela sensibilidade da região palmar desde o polegar até à face radial do $4^{\circ}$ dedo (Fig. 2), 3,7,8,10 embora existam variantes do normal, raras, que podem tornar desafiante o diagnóstico de STC. ${ }^{7}$

Na maioria dos casos a STC é idiopática. A fisiopatologia subjacente à compressão do nervo mediano é multifatorial: pode haver processos inflamatórios ou espessamento de qualquer um dos tendões que passam no canal; pode haver fibrose do tecido conjuntivo que rodeia os tendões dos flexores; o espaço anatómico do túnel cárpico pode ser mais pequeno, seja por alterações congénitas, lesões com efeito de massa (como cistos ou neoplasias), edema ou por condições inflamatórias resultantes de doenças sistémicas. ${ }^{4,7,8}$ Além disto, a postura do membro superior também influencia a pressão existente no túnel cárpico: a pressão é menor quando o punho está numa posição neutra ou em flexão ligeira, aumentando proporcionalmente com o desvio face a esta posição. $^{7}$
O aumento da pressão no canal cárpico pode lesar o nervo diretamente, prejudicar o transporte axonal ou comprimir os vasos do perineuro e causar isquemia do nervo mediano. ${ }^{9,10}$

\section{EPIDEMIOLOGIA E FATORES DE RISCO}

A STC é a mononeuropatia focal compressiva mais comum, ${ }^{1,9}$ afetando aproximadamente 3\% da população geral. ${ }^{6}$ Esta doença é frequente entre adultos, mas rara em crianças. ${ }^{7}$ Além disso, afeta mais frequentemente o sexo feminino (3:1) e a sua prevalência e gravidade aumenta com a idade. 3,6

Várias condições têm sido associadas à STC, nomeadamente: artrite e doenças do tecido conjuntivo; gravidez; obesidade; diabetes mellitus; hipotiroidismo; fraturas do punho; doença renal crónica; história familiar de STC; Exposição cumulativa a esforços, vibrações e movimentos repetitivos do punho, fatores que podem ser particularmente relevantes em determinadas profissões. ${ }^{1-6,9-11}$

\section{ABORDAGEM DIAGNÓSTICA MANIFESTACÕ̃ES CLIINICAS}

A STC refere-se ao conjunto de sinais e sintomas causados pela compressão do nervo mediano ao longo do seu trajeto no túnel cárpico. ${ }^{1-4} \bigcirc$ diagnóstico é clínico e deve ser equacionado perante sinais e sintomas característicos. ${ }^{4,12}$ 
Os sintomas clássicos da STC são dor, disestesias e/ou parestesias no território do nervo mediano (região palmar, desde o polegar até à face radial do $4^{\circ}$ dedo).,1,3 Geralmente, os sintomas sensitivos são referidos a esta região, contudo podem envolver a mão no seu todo, bem como o punho. 1,3,9 Apesar de menos frequente, poderá haver irradiação das queixas para o antebraço e, de forma mais rara, pode haver irradiação acima do nível do cotovelo, até ao ombro. 1,3,4,9

Tipicamente, o quadro clínico piora à noite, acarretando despertares noturnos e levando os utentes a agitar as mãos para tentar aliviar os sintomas (Flick sign).1,3,9 Os sintomas da STC podem também ser desencadeados por tarefas que envolvam a flexão ou extensão do punho como, por exemplo, conduzir ou costurar. . $^{3,8,9}$

Geralmente, a STC afeta ambas as mãos, embora o atingimento possa ser apenas unilateral. ${ }^{13}$ Em relação à história natural, os doentes com STC passam por períodos de remissão e exacerbação, com tendência a que os sintomas passem de intermitentes a persistentes. , $, 8,9^{-}$

Uma vez que as fibras sensitivas são mais suscetíveis à compressão do que as fibras motoras, as parestesias e a dor constituem as manifestações clínicas dominantes no início da doença. ${ }^{3}$

Em casos mais graves e arrastados, as fibras motoras acabam por ser afetadas, podendo causar fraqueza na abdução e oposição do polegar, bem como atrofia da eminência tenar.1,3,4,9 Por este motivo, os doentes podem descrever dificuldade em segurar objetos, abrir frascos ou abotoar uma camisa.,9 Quer a atrofia/fraqueza da eminência tenar, quer a diminuição/desaparecimento da dor (hipoalgesia) são manifestações tardias e indicam perda sensitiva e défice motor permanentes. ${ }^{1,3}$

\section{EXAME FÍ́SICO}

Perante suspeita de STC, o exame objetivo deve avaliar todo o membro superior, de modo a excluir outras causas que possam ser responsáveis pelo quadro clínico.3,9

Na apresentação inicial, a maioria dos doentes com STC de gravidade ligeira/moderada não apresenta alterações no exame físico. ${ }^{3}$ No entanto, a inspeção da mão e do punho pode trazer pistas sobre fatores precipitantes para esta patologia, como alterações anatómicas, alterações artríticas ou sinais sugestivos de trauma. ${ }^{3}$ Por exemplo, um pulso em forma de quadrado (com uma relação profundidade-largura aumentada) associa-se a um odds ratio de 4,56 de desenvolvimento de STC (com um intervalo de confiança de $95 \%$ entre 2,97 e 6,99). ${ }^{14}$

Deve notar-se que a sensibilidade da eminência tenar deve ser normal na STC, uma vez que provém do ramo cutâneo palmar do nervo mediano, que emerge do nervo mediano proximalmente ao túnel do carpo. ${ }^{3,8,10}$ Assim, a diminuição da sensibilidade na eminência tenar indica uma lesão do nervo mediano com origem proximal ao túnel do carpo. ${ }^{3}$

As manobras de provocação dos sintomas típicos da STC incluem os testes de Phalen, Tinel, compressão manual do carpo e elevação da mão.,12 De forma isolada, cada uma das manobras apresenta sensibilidade e especificidade limitadas. ${ }^{3}$ Contudo, são testes simples de realizar e a combinação de resultados positivos nestas manobras sugere o diagnóstico de STC. ${ }^{3}$ De seguida serão apresentadas as manobras de provocação mencionadas:

- Teste de Phalen: O utente deve manter o punho em flexão máxima durante um minuto (isto pode ser feito colocando a parte dorsal de ambas as mãos voltadas uma para a outra, com os cotovelos fletidos; em alternativa, pode ser realizada a flexão máxima do punho com o cotovelo em extensão. ${ }^{1,12}$ A manobra é considerada positiva se provocar dor e/ou parestesias no território do nervo mediano. ${ }^{1}$ Este teste apresenta uma sensibilidade de 68\% e uma especificidade de 73\%. ${ }^{15}$ Além disso, a gravidade da STC parece ser tanto maior, quanto menos tempo for necessário para que surjam sintomas. ${ }^{16}$

- Teste de Tinel: Consiste na percussão sobre o trajeto do nervo mediano no túnel cárpico. ${ }^{1,12}$ Um teste positivo é definido pelo aparecimento de dor e/ou parestesia nos dedos com inervação sensitiva proveniente do nervo mediano. ${ }^{1,12} \mathrm{~A}$ sensibilidade deste teste é cerca de $50 \%$, com uma especificidade de $77 \%{ }^{15}$

- Teste de Durkan: Realiza-se aplicando pressão sobre o ligamento transverso do carpo. É positivo se desencadear parestesias nos primeiros 30 segundos de compressão. Este teste apresenta uma sensibilidade de aproximadamente $64 \%$ e uma especificidade de $83 \% .^{15}$

- Teste da elevação das mãos: Como o nome indica, envolve levantar as mãos acima da cabeça, o que deve ser feito durante um minuto. ${ }^{3} \bigcirc$ teste é considerado positivo, se houver reprodução dos sintomas causados pela STC. ${ }^{3}$

\section{MEIOS COMPLEMENTARES DE DIAGNÓSTICO}

Como já foi referido, a STC constitui um diagnóstico clínico. ${ }^{3,4,8}$ Contudo, os testes eletrodiagnósticos (o estudo da condução nervosa e a eletromiografia) têm um papel importante na confirmação do diagnóstico e na seleção do tratamento para cada utente. ${ }^{3}$ 
O estudo da condução nervosa confirma o diagnóstico de STC quando há um atraso na velocidade de condução do estímulo nervoso no nervo mediano ao longo do seu percurso no túnel cárpico, com uma condução normal no restante trajeto. ${ }^{3}$ Esta diminuição da velocidade de condução resulta da lesão da bainha de mielina provocada pela compressão nervosa. ${ }^{12}$ Por sua vez, a eletromiografia avalia alterações patológicas nos músculos inervados pelo nervo mediano. ${ }^{3}$

Estes testes apresentam uma sensibilidade entre 56\% a $85 \%$ e uma especificidade entre $94 \%$ e $99 \%,{ }^{17}$ razão pela qual são o método mais eficaz para a confirmação do diagnóstico da STC, possibilitando ainda estratificar a gravidade desta síndrome e despistar outras patologias (polineuropatia, plexopatia e radiculopatia).,3,9 O papel confirmatório destes exames tem particular relevância

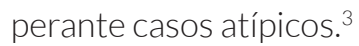

Os testes de eletrodiagnóstico permitem aferir a gravidade da STC, ajudando na seleção do tratamento mais adequado para cada situação: tratamento cirúrgico para os casos moderados/graves versus não-cirúrgico para os casos ligeiros/moderados. ${ }^{3,9}$ Além disso, dão informação prognóstica. ${ }^{3,9}$ Perante STC com lesão neurológica grave, há maior risco de: a) falência da terapêutica não-cirúrgica; b) recuperação apenas parcial mesmo com a cirurgia; b) recuperação pós-cirúrgica mais demorada. 3,9

Face ao exposto, percebe-se que os estudos de eletrodiagnóstico são os meios complementares de diagnóstico de excelência para avaliação desta síndrome. Contudo, a ecografia também pode ser útil no diagnóstico de STC,, 33 pela constatação do aumento da área do nervo mediano num corte transversal. ${ }^{3,4}$ As vantagens deste método são o seu baixo custo, o facto de ser um método não-invasivo e de permitir a avaliação de lesões com efeito de massa, tenossinovite e tendinopatias. ${ }^{3} \mathrm{~A}$ acuidade diagnóstica deste método depende do operador e das características do doente. ${ }^{12}$ Atualmente, este exame tem como desvantagens os factos de não conseguir excluir outras etiologias (como polineuropatias), nem determinar a gravidade da STC. ${ }^{3}$

Ainda no campo dos exames de imagem, a radiografia pode ser útil perante suspeita de anomalias estruturais do punho, tais como tumores, deformidades e outras patologias osteoarticulares localizadas.,.18 Por sua vez, a ressonância magnética não está geralmente recomendada. $^{3}$

Finalmente, abordando a STC num contexto clínico holístico, deve considerar-se a realização de análises laboratoriais para eventuais comorbilidades, tais como diabetes e hipotiroidismo entre outras, se existirem sinais sugestivos da presença destas doenças. ${ }^{3}$ Deve assegurar-se o estudo diagnóstico e a abordagem terapêutica de possíveis comorbilidades associadas à STC, embora não haja evidência de que o tratamento dessas patologias acarrete melhoria dos sintomas ou da história natural da STC. ${ }^{19}$

\section{ABORDAGEM TERAPÊUTICA}

As estratégias terapêuticas para a STC englobam as medidas conservadoras (não-cirúrgicas) e o tratamento cirúrgico. ${ }^{9}$ A estratégia mais adequada para cada situação dependerá da gravidade da doença. ${ }^{3}$

Nos casos de doença ligeira/moderada, sem evidência de perda axonal ou desenervação nos estudos eletrodiagnósticos, recomenda-se o tratamento inicial com medidas não-cirúrgicas. ${ }^{3,5}$

Perante quadros moderados/graves, com lesão neurológica confirmada nos estudos eletrodiagnósticos, deve proceder-se à referenciação do utente para intervenção cirúrgica, exceto no caso de existir um fator precipitante, tal como a gravidez. ${ }^{3,5,19}$

A STC pode surgir durante a gravidez, principalmente no terceiro trimestre. ${ }^{19}$ Na maioria dos casos, os sintomas têm remissão espontânea algumas semanas após o parto. ${ }^{19} \mathrm{O}$ tratamento de primeira linha neste contexto consiste no uso de uma ortótese de imobilização do punho.,19 Raramente está indicada a descompressão cirúrgica, atendendo ao caráter autolimitado das queixas no contexto da gravidez. ${ }^{19}$

\section{TRATAMENTO CONSERVADOR}

As medidas de tratamento conservador incluem: ortótese de imobilização do punho; glicocorticoides por via oral ou por via injetável tópica; fisioterapia; ioga.,19

Geralmente, as medidas de tratamento conservador permitem uma melhoria dos sintomas no prazo de duas a seis semanas, atingindo o benefício máximo em três meses. ${ }^{20}$ Não havendo melhoria dos sintomas após seis semanas, deve adotar-se uma abordagem terapêutica diferente. ${ }^{3}$

De seguida apresentam-se fatores preditores de falência do tratamento conservador: Longa duração dos sintomas (mais de 6-12 meses); Idade superior a 50 anos; Parestesia constante; Discriminação de dois pontos prejudicada (> $6 \mathrm{~mm}$ ); Teste de Phalen positivo em menos de 30 segundos; Latências motoras e sensitivas aumentadas, demonstradas por testes de eletrodiagnóstico. ${ }^{19}$

\section{Ortótese de imobilização do punho}

A ortótese mantém uma posição neutra, prevenindo a flexão ou a extensão do punho de forma prolongada. ${ }^{19}$ 
Geralmente, as ortóteses são usadas durante a noite, mas podem ser usadas de forma contínua ao longo de todo o dia. ${ }^{19}$ Por si só, o uso de ortótese pode reduzir a intensidade dos sintomas, associar-se a uma melhoria da velocidade de condução no nervo mediano, bem como atrasar ou eliminar a necessidade de intervenção cirúrgica. ${ }^{21-23}$

O uso de ortótese é o tratamento de primeira linha para a STC ligeira/moderada, devido à simplicidade do seu uso, baixo custo e boa tolerabilidade. ${ }^{3}$ Além disso, o uso de ortóteses pode ser conjugado com outras modalidades de tratamento. ${ }^{3}$

\section{Injeção local de glicocorticoides}

A injeção de glicocorticoides na região do túnel cárpico tem como objetivo reduzir a inflamação tecidual, melhorando assim os sintomas. ${ }^{4,19}$ Esta opção constitui uma alternativa, ou mesmo um complemento, ao uso de ortóteses, nos utentes recetivos a uma opção minimamente invasiva para obtenção de alívio dos sintomas a curto prazo. ${ }^{19}$

Existe cada vez mais evidência do benefício da injeção local de glicocorticoides para o tratamento da STC. ${ }^{3}$ Geralmente, esta intervenção reduz os sintomas durante um a três meses, em comparação com o placebo. ${ }^{3,18}$ Contudo, o benefício pode estender-se para além deste período, permitindo atrasar em cerca de um ano a necessidade de intervenção cirúrgica. ${ }^{3}$

Relativamente a este procedimento, não existe evidência de superioridade de uma determinada técnica de injeção ou de uma formulação específica de glicocorticoide. ${ }^{3}$ Embora esta manobra seja geralmente segura, é necessário ter em consideração o risco de lesão do nervo mediano e rutura tendinosa. ${ }^{3}$

Este procedimento pode ser repetido no mesmo punho, com seis meses de intervalo. ${ }^{3}$ Contudo, se houver recorrência dos sintomas depois de duas injeções no mesmo punho, deve considerar-se outras medidas de tratamento, cirúrgicas ou não-cirúrgicas. 3,19 De facto, até 50\% dos utentes pode ter recorrência dos sintomas depois de um período de melhoria inicial, em contraste com o que se verifica com a cirurgia (em que recorrência após um ano do procedimento é rara). ${ }^{8}$

\section{Glicocorticoides por via oral}

Em comparação com o placebo, a administração de 20 mg de prednisolona por via oral, durante 10 a 14 dias, associa-se a uma melhoria dos sintomas e funcionalidade, efeito este que pode durar até oito semanas. ${ }^{3}$ Contudo, a administração de glicocorticoides por via oral parece ser menos eficaz do que a injeção local destes fármacos. ${ }^{3}$

Outros fármacos, tais como anti-inflamatórios não-es- teroides, vitamina B6 e diuréticos, não parecem ser eficazes para o alívio sintomático nos doentes com STC.3,19

\section{Outras medidas de tratamento conservador}

A evidência é escassa no que diz respeito ao benefício das técnicas de fisioterapia (tais como mobilização dos ossos cárpicos, ultrassons com fins terapêuticos e exercícios de deslizamento do nervo mediano) na STC.3,19 Sobre o efeito do loga, a evidência é escassa e de qualidade limitada, contudo, se estiver disponível, pode tentar-se esta intervenção terapêutica. ${ }^{19}$

\section{TRATAMENTO CIRÚRGICO}

Não havendo melhoria após várias semanas desde o início do tratamento conservador, deve ponderar-se a referenciação para tratamento cirúrgico. ${ }^{8}$ Além disso, a cirurgia é o tratamento de eleição perante quadros moderados/graves, com lesão neurológica confirmada nos estudos eletrodiagnósticos. 3,5,19

A cirurgia é a intervenção com melhores resultados a médio e longo prazo. ${ }^{24}$ De facto, a descompressão cirúrgica consegue resultados satisfatórios e duradouros em $70 \%$ a $90 \%$ dos casos. ${ }^{25}$

O tratamento cirúrgico consiste na libertação do conteúdo do túnel cárpico através da secção do ligamento transverso do carpo. ${ }^{4}$ Este tratamento pode ser realizado: a) pela técnica "tradicional", aberta (incisão longitudinal longa no punho, com visualização direta do ligamento), atualmente em desuso; b) através de uma abordagem minimamente invasiva (incisão curta no punho), a mais utilizada atualmente; ou c) através de uma técnica endoscópica. ${ }^{4}$ A abordagem endoscópica apresenta maior taxa de complicações, nomeadamente lesão do nervo mediano e libertação incompleta do túnel cárpico. $^{26}$

A maioria dos doentes nota uma melhoria significativa dos sintomas dentro de uma semana após a cirurgia, conseguindo retomar a sua atividade profissional em duas semanas. ${ }^{3}$ Contudo, em alguns casos, especialmente perante STC grave, pode demorar um ano até que se atinja a remissão completa das queixas. ${ }^{3}$

A complicação pós-operatória mais frequente é a dor na cicatriz e na zona adjacente ao local da secção do ligamento transverso. ${ }^{3}$

\section{Falência do tratamento cirúrgico}

A persistência dos sintomas após a cirurgia pode dever-se, entre outros, a fibrose circunferencial ou a uma libertação incompleta do ligamento transverso do carpo. ${ }^{19}$ Nestes casos, pode haver indicação para revisão cirúrgica. 


\section{CONCLUSÃO}

A STC é uma patologia prevalente na população geral, sendo responsável por uma diminuição da qualidade de vida e por consequências nefastas no setor económico e da saúde.

Perante dor, disestesias e/ou parestesias no território do nervo mediano que pioram à noite, o médico deve equacionar este diagnóstico e recorrer às provas provocatórias. Os testes eletrodiagnósticos são o método mais eficaz para confirmação do diagnóstico, estratificação de gravidade e despiste de outras patologias.

O tratamento mais adequado para cada situação dependerá da gravidade da doença. Perante doença ligeira/ moderada recomenda-se tratamento inicial com medidas não-cirúrgicas. Perante quadros graves, está recomendado a cirurgia que, de facto, é a intervenção com melhores resultados a médio e longo prazo.

\section{RESPONSABILIDADES ÉTICAS}

CONFLITOS DE INTERESSE: Os autores declaram não possuir conflitos de interesse.

SUPORTE FINANCEIRO: O presente trabalho não foi suportado por nenhum subsídio ou bolsa.

PROVENIÊNCIA E REVISÃO POR PARES: Não comissionado; revisão externa por pares.

\section{ETHICAL DISCLOSURES}

CONFLICTS OF INTEREST: The authors have no conflicts of interest to declare.

FINANCIAL SUPPORT: This work has not received any contribution grant or scholarship.

PROVENANCE AND PEER REVIEW: Not commissioned; externally peer reviewed.

\section{REFERÊNCIAS}

1. Cardoso A, Branco J, Silva J, Cruz M, Costa M. Síndrome do túnel cárpico. In: Cardoso A, Branco J, Silva J, Cruz M, Costa M. Regras de ouro em reumatologia. Lisboa: Direção-Geral da Saúde; 2005. P. 58-60.

2. Costa M, Branco J. Dor no punho e/ou mão. In: Costa M, Branco J. Reumatologia Básica em Medicina Geral e Familiar. Lisboa: LIDEL; 2019.p. 194-205.

3. Wipperman J, Goerl K. Carpal tunnel syndrome: diagnosis and management. Am Fam Physician. 2016;94:993-9.

4. Padua L, Coraci D, Erra C, Pazzaglia C, Paolasso I, Loreti C, et al. Carpal tunnel syndrome: clinical features, diagnosis, and management. Lancet Neurol. 2016;15:1273-84. doi: 10.1016/ S1474-4422(16)30231-9.
5. Wieland LS. Acupuncture and related interventions for the treatment of symptoms associated with carpal tunnel syndrome: Summary of a Cochrane Review. Explore. 2019;15:243-4. doi: 10.1016/j.explore.2019.02.008.

6. Pourmemari MH, Heliövaara M, Viikari-Juntura E, Shiri R. Carpal tunnel release: Lifetime prevalence, annual incidence, and risk factors. Muscle Nerve. 2018;58:497-502. doi: 10.1002/ mus.26145.

7. Kothari M. Carpal tunnel syndrome: Etiology and epidemiology. In: UpToDate, Post TW (Ed), UpToDate, Waltham, MA [Acedido a 31 de janeiro de 2021]. Disponível em: https:// www.uptodate.com/contents/carpal-tunnel-syndrome-etiology-and-epidemiology.

8. Doughty CT, Bowley MP. Entrapment Neuropathies of the Upper Extremity. Med Clin North Am. 2019;103:357-70.

9. Wang L. Guiding treatment for carpal tunnel syndrome. Phys Med Rehabil Clin N Am. 2018;29:751-60. doi: 10.1016/j. mcna.2018.10.012.

10. Aboonq MS. Pathophysiology of carpal tunnel syndrome. Neurosciences. 2015;20:4-9.

11. Kozak A, Schedlbauer G, Wirth T, Euler U, Westermann C, Nienhaus A. Association between work-related biomechanical risk factors and the occurrence of carpal tunnel syndrome: an overview of systematic reviews and a meta-analysis of current research. BMC Musculoskelet Disord. 2015;16:231. doi: 10.1186/s12891-015-0685-0.

12. Kothari M. Carpal tunnel syndrome: Clinical manifestations and diagnosis. In: UpToDate, Post TW (Ed), UpToDate, Waltham, MA [Acedido a 31 de janeiro de 2021]. Disponível em: https://www.uptodate.com/contents/carpal-tunnel-syndrome-clinical-manifestations-and-diagnosis.

13. Dec P, Zyluk A. Bilateral carpal tunnel syndrome - A review. Neurol Neurochir Pol. 2018;52:79-83.

14. Shiri R. A square-shaped wrist as a predictor of carpal tunnel syndrome: A meta-analysis. Muscle Nerve. 2015;52:709-13. doi: 10.1002/mus.24761.

15. MacDermid JC, Wessel J. Clinical diagnosis of carpal tunnel syndrome: a systematic review. J Hand Ther. 2004;17:309-19. doi: 10.1197/j.jht.2004.02.015.

16. Priganc VW, Henry SM. The relationship among five common carpal tunnel syndrome tests and the severity of carpal tunnel syndrome. J Hand Ther. 2003;16:225-36.

17. Jablecki CK, Andary MT, Floeter MK, Miller RG, Quartly CA, Vennix MJ, et al. Practice parameter: Electrodiagnostic studies in carpal tunnel syndrome. Report of the American Association of Electrodiagnostic Medicine, American Academy of Neurology, and the American Academy of Physical Medicine and Rehabilitation. Neurology. 2002;58:1589-92.

18. Kleopa KA. In the Clinic. Carpal tunnel syndrome. Ann Intern Med. 2015;163:Itc1. doi: 10.7326/AITC201509010.

19. Kothari M. Carpal tunnel syndrome: Treatment and prognosis. In: UpToDate, Post TW (Ed), UpToDate, Waltham, MA [Acedido a 31 de janeiro de 2021]. Disponível em: https://www. uptodate.com/contents/carpal-tunnel-syndrome-treatment-and-prognosis.

20.Shi Q, MacDermid JC. Is surgical intervention more effective than non-surgical treatment for carpal tunnel syndrome? A systematic review. J Orthop Surg Res. 2011;6:17. doi: 10.1186/1749-799X-6-17.

21. Muller M, Tsui D, Schnurr R, Biddulph-Deisroth L, Hard J, MacDermid JC. Effectiveness of hand therapy interventions in primary management of carpal tunnel syndrome: a systematic review. J Hand Ther. 2004;17:210-28. 
22. Manente G, Torrieri F, Di Blasio F, Staniscia T, Romano F, Uncini A. An innovative hand brace for carpal tunnel syndrome: a randomized controlled trial. Muscle Nerve. 2001;24:1020-5.

23. Sevim S, Dogu O, Camdeviren H, Kaleagasi H, Aral M, Arslan E, et al. Long-term effectiveness of steroid injections and splinting in mild and moderate carpal tunnel syndrome. Neurol Sci. 2004;25:48-52.

24. Huisstede BM, Hoogvliet P, Franke TP, Randsdorp MS, Koes BW. Carpal tunnel syndrome: effectiveness of physical therapy and electrophysical modalities. An Updated Systematic Review of Randomized Controlled Trials. Arch Phys Med Rehabil. 2018;99:1623-34.e23. doi: 10.1016/j.apmr.2017.08.482.

25. Turner A, Kimble F, Gulyás K, Ball J. Can the outcome of open carpal tunnel release be predicted?: a review of the literature. ANZ J Surg. 2010;80:50-4.

26. Vasiliadis HS, Nikolakopoulou A, Shrier I, Lunn MP, Brassington R, Scholten RJ, et al. Endoscopic and open release similarly safe for the treatment of carpal tunnel syndrome. a systematic review and meta-analysis. PLoS One. 2015;10:e0143683. doi: 10.1371/journal.pone.0143683. 